\title{
Oral Lichen Planus Developing after PD-1 Inhibitor Therapy in Two Patients with Malignant Melanoma
}

\begin{abstract}
Cutaneous adverse reactions to checkpoint inhibitor therapy commonly include non-specific dermatitis and pruritus, though the development of a wide range of dermatologic toxicities has been described, including lichenoid reactions. Occasional oral mucosal involvement has been reported, with rare erosive changes. Here, we present two cases of erosive oral lichen planus developing after PD-1 inhibitor therapy for malignant melanoma, with one case that later progressed to diffuse cutaneous involvement. Oral involvement in both cases was treated with topical high potency steroids and topical tacrolimus. This treatment regimen allowed one patient to continue immunotherapy and the other to resume immunotherapy after it was discontinued. These cases support erosive oral lichen planus as a notable, serious adverse effect of PD-1 inhibitors.
\end{abstract}

\section{Introduction}

Advances in immunotherapy, including the programmed death 1 (PD-1) checkpoint inhibitors nivolumab and pembrolizumab, have considerably changed the management of advanced malignant melanoma. These monoclonal antibodies impede binding of PD-1 with its ligand, PD-L1, preventing T-cell apoptosis and stimulating antitumor response [1]. Cutaneous immune-related adverse effects from PD-1 inhibitor therapy occur frequently, with non-specific rash and pruritus among the most commonly reported $[2,3]$. The extent of their cutaneous toxicities, however, has yet to be fully identified. Here, we present two patients with advanced malignant melanoma who underwent adjuvant therapy with nivolumab and later presented with erosive oral lichen planus.

\section{Case Presentation}

\section{Case 1}

A 78-year-old female with a history of Diffuse Large B-Cell Lymphoma (DLBCL) and malignant melanoma presented to dermatology clinic for painful erosive lesions on her lower lip following two months of therapy with nivolumab. She was initially diagnosed with a stage T2a melanoma of her back in March 2015 and underwent a $2 \mathrm{~cm}$ wide local excision with negative sentinel lymph node biopsy. Surveillance imaging and subsequent biopsy in March 2016 revealed metastatic melanoma in the lung, treated with wedge resection. In February 2017, surveillance MRI detected a subcutaneous nodule of the posterior trunk; pathology confirmed metastatic disease. After surgical excision of the nodule, the patient was started on high-dose ipilimumab in May 2017. While on ipilimumab, the patient developed new foci of disease with subcutaneous nodules on the back and later on the abdomen. Both lesions were resected.

\section{Clinical \& Investigative Dermatology}

Jain $\mathbf{N}^{1}$, Panah $\mathrm{E}^{1}$, Garfield $\mathrm{E}^{1}$, Quan $\mathrm{V}^{1}$, Shi $\mathrm{K}^{1}$, Mohan L1, Yoo $\mathbf{S}^{1}$, Chandra $\mathbf{S}^{2}$, Sosman $\mathbf{J}^{2}$ and Gerami $\mathbf{P}^{1^{*}}$

${ }^{\prime}$ Department of Dermatology, Northwestern University Feinberg School of Medicine, USA

${ }^{2}$ Division of Hematology and Oncology, Northwestern University Feinberg School of Medicine, USA

\section{*Address for Correspondence}

Gerami P, Northwestern University, Department of Dermatology, 676 N. St Clair St., Suite 1600 Chicago, IL, USA, Tel: (312) 695-1413; Email: p-gerami@northwestern.edu

\section{Submission: 30 May, 2019}

Accepted: 19 June, 2019

Published: 28 June, 2019

Copyright: (๑) 2019 Jain N, et al. This is an open access article distributed under the Creative Commons Attribution License, which permits unrestricted use, distribution, and reproduction in any medium, provided the original work is properly cited.

Ipilimumab was discontinued, and she was started on nivolumab in January 2018. After two months of treatment with nivolumab, the patient presented to the emergency department with progressive lower lip ulcerations and gingival erosions associated with burning, bleeding, and difficulty tolerating oral intake secondary to pain. She was initially treated with valacyclovir without improvement. Viral culture was negative for herpes simplex viruses $1 \& 2$. She was referred to dermatology for further evaluation. On physical examination, an extensive $4 \mathrm{~cm}$ heme-crusted tender ulceration was noted on the lower vermilion lip (Figure 1). Punch biopsy revealed erosive lichen planus (Figure 2). Nivolumab was temporarily held, and the patient was treated with topical clobetasol $0.05 \%$ ointment and bacitracinpolymyxin ointment twice daily for two weeks followed by topical tacrolimus $0.1 \%$ ointment twice daily. Significant improvement in the lesion was noted after one month of treatment (Figure 3). The patient resumed immunotherapy after 4 weeks, and her oral lichen planus remained stabilized with topical therapy. She subsequently developed bullous lesions on her bilateral shins and feet. Pathology demonstrated a lichenoid process, and direct immunofluorescence studies were negative for immune deposits.

Case 2

A 70-year-old male with a history of psoriasis was referred to dermatology after diagnosis of a T4bN1aM0 melanoma of the right arm, status post wide local excision and axillary sentinel lymph node

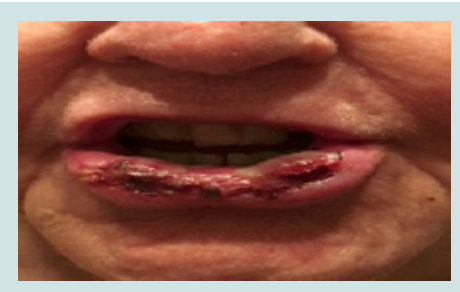

Figure 1: Case 1- clinical presentation prior to treatment. 


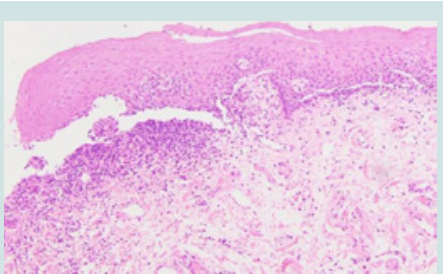

Figure 2: Case 1- hematoxylin and eosin stain showing a lichenoid infiltrate with squamatization of the basal layer, consistent with lichen planus.

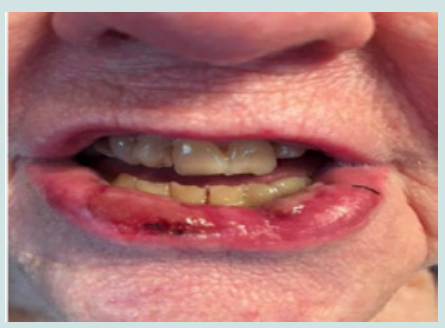

Figure 3: Case 1- clinical presentation after treatment.

biopsy. One of four lymph nodes was positive for melanoma, and the patient underwent a complete right axillary lymph node dissection with no additional positive lymph nodes. PET/CT and MRI scans did not show evidence of metastatic disease. The patient was enrolled in a clinical trial of adjuvant immunotherapy randomized to nivolumab or combination ipilimumab/nivolumab in February 2018. Two months after starting treatment, the patient presented to his oncologist with a lip ulceration, which was initially treated with topical acyclovir without improvement. Evaluation by a dermatologist revealed 2 $\mathrm{cm}$ heme-crusted erosion on the lower vermilion lip. Punch biopsy was consistent with oral lichen planus. The patient was started on topical clobetasol $0.05 \%$ ointment twice daily for one week followed by alternating topical tacrolimus $0.1 \%$ ointment twice daily MondayThursday with clobetasol $0.05 \%$ ointment twice daily FridaySunday, with notable improvement of the lesion within 3-4 weeks. Immunotherapy was continued while his oral lichen planus was treated.

\section{Discussion}

Lichen planus is a $\mathrm{CD} 8^{+} \mathrm{T}$-cell mediated autoimmune process targeting basal keratinocytes causing apoptosis of epithelial cells and resultant erosions and ulceration of mucocutaneous sites. The precise mechanism of drug-induced lichenoid reactions is not completely understood, but likely involves breaking the normal tolerance of $\mathrm{CD}^{+}$lymphocytes for the epithelium. One signaling pathway which may induce tolerance for the epithelium is through the interaction of PD-1 with ligand B7-H1 [4]. Blockade of this interaction by pembrolizumab and nivolumab likely tips the delicate balance of tolerance allowing $\mathrm{CD}^{+}$lymphocytes to react against epithelial cells. A number of other medications have been also associated with oral lichenoid reactions, including angiotensin-converting enzyme inhibitors, anticonvulsants, antiretrovirals, nonsteroidal anti-inflammatory drugs, and more recently TNF-alpha and tyrosine kinase inhibitors, though the pathogenesis is likely different than that of checkpoint inhibitors $[5,6]$.

Several cutaneous adverse effects of PD-1 inhibitors have been described, however, literature detailing oral mucosal toxicity and optimal treatment strategies for this toxicity in patients treated with checkpoint inhibitors for melanoma is more limited. Of lichenoid eruptions $[7,8]$, one study found mucosal involvement in only 1 of 14 patients [8]. Here we present two patients with advanced melanoma who developed oral lichen planus within two months of treatment with nivolumab. Both patients initially presented with isolated oral involvement. In our first patient, the extensive nature of and symptoms from her lichen planus led to temporary discontinuation of nivolumab. The second patient did not require holding of immunotherapy. Both patients improved with very high potency topical steroids and topical calcineurin inhibitors, allowing the first patient to resume nivolumab. Oral glucocorticoids or systemic steroid-sparing immunosuppressive agents were not used.

\section{Conclusion}

Our cases support considering oral lichen planus as a notable cutaneous adverse event in patients receiving PD-1 inhibitor therapy for the treatment of melanoma. Recognition of erosive lichen planus early as an adverse event by both oncologists and dermatologists may potentially avoid disruptions in immunotherapy with appropriate treatment. Also, this experience suggests that topical therapy alone either with high potency topical steroids or in combination with tacrolimus ointment can provide a sustained response while immunotherapy is continued. Of note, both patients had comorbidities (DLBCL and psoriasis) suggestive of baseline immune dysregulation. Future research investigating specific cutaneous adverse effects of checkpoint inhibitors can yield more definitive answers about their association with development of other immune-mediated adverse reactions and implications for tumor response to therapy.

\section{References}

1. Wakade DV, Carlos G, Hwang SJ, Chou S, Hui R, et al. (2016) PD-1 inhibitors induced bullous lichen planus-like reactions: a rare presentation and report of three cases. Melanoma Res 26: 421-424.

2. Owosho AA, Randazzo J, Rosen EB, Estilo CL, Huryn JM, et al. (2016) Squamous cell carcinoma associated with chronic graft versus host diseaselike/lichen planus-like lesion of the oral cavity in a patient managed for metastatic melanoma with a PD-1 inhibitor pembrolizumab. Oral Oncol 63 e1-e3.

3. Brahmer JR, Tykodi SS, Chow LQ, Hwu WJ, Topalian SL, et al. (2012) Safety and activity of anti-PD-L1 antibody in patients with advanced cancer. N Engl J Med 366: 2455-2465

4. Zhou G, Zhang J, Ren XW, Hu JY, Du GF, et al. (2012) Increased B7-H1 expression on peripheral blood $\mathrm{T}$ cells in oral lichen planus correlated with disease severity. J Clin Immunol 32: 794-801.

5. Woo V, Bonks J, Borukhova L, Zegarelli D (2009) Oral lichenoid drug eruption: a report of a pediatric case and review of the literature. Pediatr Dermatol 26 : 458-64.

6. Asarch A, Gottlieb AB, Lee J, Masterpol KS, Scheinman PL, et al (2009) Lichen planus-like eruptions: an emerging side effect of tumor necrosis factor-alpha antagonists. J Am Acad Dermatol 61: 104-111.

7. Shi VJ, Rodic N, Gettinger S, Leventhal JS, Neckman JP, et al. (2016) Clinical and histologic features of lichenoid mucocutaneous eruptions due to anti-programmed cell death 1 and anti-programmed cell death ligand 1 immunotherapy. JAMA dermatology 152: 1128-1136.

8. Hwang SJ, Carlos G, Wakade D, Byth K, Kong BY, et al. (2016) Cutaneous adverse events (AEs) of anti-programmed cell death (PD)-1 therapy in patients with metastatic melanoma: A single-institution cohort. J Am Acad Dermatol. 74: 455-61 e1. 